\title{
Análisis de los registros en Moodle para estudiar la actividad desarrollada por estudiantes de Histología Humana y su relación con el rendimiento académico
}

\author{
María Pilar Álvarez Vázquez ${ }^{a}$, María Teresa Angulo Carrere ${ }^{b}$, María Carmen Bravo Llatas ${ }^{\mathfrak{c}}$, Jesús \\ Cristóbal Barrios ${ }^{\mathrm{d}}$ y Ana María Álvarez-Méndez \\ aSección departamental de Biología Celular, Facultad de Medicina, UCM, pilar@med.ucm.es, bDepartamento de \\ Enfermería, Facultad de Enfermería, Fisioterapia y Podología, UCM, anguloca@enf.ucm.es y amalvare@ucm.es, ${ }^{c}$ Área \\ de Gobierno de Tecnologías de la Información y de Apoyo Técnico al Usuario, UCM, mcbravo@ucm.es y dérea de \\ Software Corporativo, UCM, jcristobal@ucm.es
}

\begin{abstract}
This paper presents the results obtained after extracting and processing the logs stored in Moodle platform corresponding to the activity of human histology' students in the virtual campus. Data were analysed with the softwares $R$ and SPSS to reveal students' behavior and possible differences among them according to their academic performance were studied. Results showed a huge activity of the students much higher than the mean of Medicine virtual campuses and that the activity is clearly dependent of the academic schedule. Student's behavior was not the same but, on the contrary, differences were pointed out when comparing clusters according to the theory grade. The decision tree revealed that students who passed with continuous assessment and those who must do a final exam have made a statistically different use of the virtual campus.
\end{abstract}

Keywords: human histology, moodle, logs, students' behavior, academic performance

\section{Resumen}

Este trabajo presenta los resultados obtenidos tras la extracción y análisis de los registros almacenados en Moodle correspondientes a la actividad desarrollada por los estudiantes de histología humana en el campus virtual. Mediante el lenguaje de programación $R$ y el programa SPSS se estudió el comportamiento de los alumnos y las posibles diferencias entre estudiantes según su rendimiento académico. Los datos revelan que los estudiantes entran masivamente al campus virtual, con una actividad muy superior a la media de la titulación, y claramente dependiente del calendario académico. Su comportamiento no es homogéneo, observándose diferencias entre los grupos de alumnos según su nota final de teoría. El árbol de decisión obtenido al contrastar el uso efectuado por estudiantes que aprobaron por parciales y los aprobados en el examen final permite establecer diferencias estadisticamente significativas.

Palabras clave: histología humana, moodle, registros, comportamiento de los estudiantes, rendimiento académico

\section{Introducción}

La Histología humana es una ciencia morfológica que estudia con el microscopio las características de células, matrices extracelulares, tejidos y órganos humanos, situándose entre las ciencias que se ocupan de 
niveles organizativos inferiores (Biología molecular) y superiores (Anatomía). Forma parte del grupo de ciencias básicas que se incluyen en los planes de estudio de medicina de las universidades y es la base sobre la que se sustenta la Anatomía Patológica, especialidad clínica imprescindible en el diagnóstico y pronóstico de enfermedades mediante el estudio de biopsias, piezas quirúrgicas, citologías y autopsias.

En el Grado en Medicina de la Universidad Complutense de Madrid (UCM), la histología se estudia en primer curso dentro de la asignatura Biología celular, Embriología general e Histología humana y, en segundo curso, dentro de la Organografia microscópica humana $(\mathrm{OMH})$. En ambas materias la docencia gira en torno a clases magistrales y sesiones de prácticas con microscopio. El aprendizaje de su amplio contenido curricular, en buena parte basado en la memoria, convertía tradicionalmente a la OMH en un reto importante para el alumnado de segundo curso. La evaluación, con un único examen final, presentaba un alta tasa de suspensos, si bien es cierto que en la convocatoria extraordinaria (septiembre) un porcentaje importante de estudiantes lograba superarlo. La reforma curricular del plan Bolonia supuso en el caso de la $\mathrm{OMH}$ una importante reducción de horas, sobre todo de sesiones de prácticas, la introducción del llamado trabajo no presencial y la planificación conjunta de la asignatura con otras dos, Anatomía Humana (AH) y Fisiología Humana (FH) dentro del módulo Estructura y función del cuerpo humano en segundo curso (UCM, 2019). De esta forma, los tres programas teóricos se organizaron en bloques, con un examen parcial al término de cada uno, con lo que los estudiantes se examinarían de las tres asignaturas de forma consecutiva en el mismo día. Poco tiempo después de la implantación del plan Bolonia, el grado en Medicina también modificó su calendario académico de forma que la convocatoria extraordinaria pasó a ser antes de las vacaciones de verano (julio).

En las últimas décadas los profesores universitarios han visto crecer sus funciones y tareas docentes. Ya no solo preparan e imparten clases teóricas y prácticas, preparan y corrigen exámenes, sino que han ampliado y diversificado sus metodologías docentes (aprendizaje mediante resolución de casos y de problemas, mediante proyectos, aula inversa, gamificación, simulaciones, microscopios virtuales, grupos pequeños de trabajo, etc.) y han adoptado nuevos roles (Salinas, 2004; Zambrano et al., 2010; Mas, 2011). Todo esto ha supuesto un mayor interés por la formación continua y un incremento en la dedicación y el esfuerzo. La irrupción de las tecnologías de información y la comunicación (TIC) en la docencia universitaria ha permitido que el profesorado y el alumnado tengan nuevos espacios de trabajo, más allá de las aulas físicas. Las instituciones de educación superior y las universidades han incorporado masivamente sistemas de gestión del aprendizaje (LMS por Learning Management System en inglés) siendo la plataforma Moodle (Dynamic learning environment oriented to modular objects) la de mayor implantación. Moodle se inspira en la pedagogía constructivista y proporciona un entorno virtual que favorece la interacción entre participantes y el aprendizaje (Paulsen, 2003). En el llamado campus virtual (CV) cada profesor puede elegir una serie de herramientas para facilitar el aprendizaje de sus estudiantes y subir materiales y recursos propios o enlazar con otros que considere de utilidad. De esta forma, las TIC se han convertido en un apoyo imprescindible para docentes y estudiantes. No obstante, la incorporación de las TIC no debería servir para seguir haciendo lo mismo con nuevas herramientas sino para hacer cosas nuevas de modos nuevos (Llorens, 2014) y favorecer el aprendizaje activo, esto es, autónomo, progresivo, interactivo y personalizado (GarcíaPeñalvo et al., 2015).

Las interacciones que se producen cuando un participante accede a un espacio virtualizado en Moodle se conservan en la propia plataforma y pueden constituir una fuente de información valiosa. El desarrollo de técnicas de análisis masivo de datos (Data mining) ha hecho posible extraer y procesar información valiosa 
(Mayer-Schönberger y Cukier, 2013; Daniel, 2015). Sin embargo, bien por falta de conocimientos y de recursos bien por la carga de trabajo excesiva, los docentes no acceden a los datos almacenados (registros o $\log s$ ), por lo que la inmensa mayoría ignora la información contenida en ellos. Analizar el rastro que dejan los participantes en un espacio digital no solo permite conocer con detalle el uso que han hecho de los recursos y herramientas programadas, sino también si existen distintos clústeres o grupos de estudiantes con comportamientos diferenciados. Más allá de un análisis descriptivo del comportamiento de los estudiantes, resultaría de interés relacionar las tipologías de estudiantes en el CV con su rendimiento o, a la inversa, relacionar categorías de estudiantes según sus calificaciones con el uso hecho en el CV, pues se podrían revelar patrones de uso que permitieran predecir el rendimiento y sirvieran para la toma de decisiones de cara a cursos sucesivos (Casey y Gibson, 2010; Chaparro et al., 2010; Long et al., 2011; Romero et al., 2013; Torres-Porras et al., 2018). Así mismo sería pertinente informar al alumnado de las evidencias halladas, es decir, cómo el comportamiento en el espacio digital se relaciona con el rendimiento.

Este trabajo forma parte del proyecto de innovación y mejora de la calidad docente titulado "Lo que no conocemos de nuestros estudiantes de CC de la Salud y nos revelan los rastros digitales. Learning Analytics: herramienta con potencial transformador para mejorar nuestra docencia” concedido por el Vicerrectorado de Calidad de la UCM para el curso 2018/19.

\section{Objetivos}

El objetivo de este trabajo fue doble. En primer lugar, estudiar el comportamiento en el CV de los estudiantes de la asignatura OMH en el curso 2018/19, esto es, describir el uso que hacen de recursos y herramientas facilitadas a través del CV. En segundo lugar, averiguar si existen relaciones entre el uso del $\mathrm{CV}$ y su rendimiento académico. En la actual tendencia de disminución de horas de clases presenciales y de potenciar el aprendizaje autónomo y a distancia, conocer en qué medida el CV influye en el rendimiento, saber si el alumnado aprovecha adecuadamente los materiales y herramientas diseñadas específicamente para cada asignatura, puede ser un indicador de la marcha académica en general y alertar de la existencia de alumnos en riesgo de abandono o de fracaso.

\section{Desarrollo de la innovación}

$O M H$ es una asignatura obligatoria, con 9 ECTS, impartida en el segundo curso del grado en Medicina en la UCM. Su calendario comienza a primeros de septiembre y acaba a mediados de marzo, si bien el examen final se programa en junio y, en segunda convocatoria, en julio. La parte teórica se organiza en 5 bloques y para su evaluación se programan 5 exámenes parciales y un examen final, del que quedan exentos los estudiantes que cumplan determinados requisitos. De cara a favorecer la implicación del estudiante, se tienen en cuenta en la nota final de cada bloque su participación en actividades realizadas en clase (casos clínicos y mapas conceptuales) y en el CV (cuestionarios y foro general).

La profesora virtualiza la asignatura en la plataforma Moodle al inicio del curso. El espacio digital, con código 18-93062, y en adelante asignatura virtualizada, permite no solo facilitar archivos informativos (guía 
docente, horarios de tutorías, normativa...), recursos (presentaciones, bibliografía recomendada....) у enlaces a páginas web (URLs), sino también ofertar herramientas para el aprendizaje (59 cuestionarios a lo largo de los bloques para estudiar y autoevaluarse antes de cada examen parcial, y un foro general para plantear/resolver dudas sobre contenidos) y la comunicación (foro de avisos, correo y mensajería). Adicionalmente, la profesora virtualizó un segundo espacio dedicado a la parte práctica, el cual no es objeto de la presente comunicación.

El curso 2018/19 el grupo estuvo formado por 95 estudiantes. Para llevar a cabo este estudio se extrajeron los registros almacenados en la asignatura virtualizada en Moodle. Un total de 171.599 registros se depuraron, anonimizaron, procesaron y estandarizaron, excluyendo aquellos que no correspondieran a participantes con el rol de estudiantes. Los registros fueron analizados con el lenguaje de programación $\mathrm{R}$ y con RStudio, entorno de desarrollo integrado para R. El software R es de uso libre, gratuito, de acceso abierto, muy versátil y potente, y es ampliamente utilizado en el entorno universitario. Tiene la posibilidad de cargar diferentes bibliotecas o paquetes con funcionalidades de cálculo y gráficas, pudiendo así realizar análisis muy sofisticados (Ferrero y López, 2018; Ollé, 2019). Se estudiaron el número de visitas diarias, la frecuencia de accesos a recursos y URLs, el acceso a cuestionarios y a foros. Los datos relativos al rendimiento académico fueron facilitados por la profesora encargada de la asignatura y tratados de forma anonimizada. El alumnado se segregó en 4 categorías de acuerdo a la nota final de la parte teórica. Los tramos fueron los siguientes: suspensos, con notas inferiores a 5; aprobados, con notas entre 5 y 6,94; notables, con calificaciones entre 6,95 y 8,54; y sobresalientes, con notas a partir de 8,5. Los resultados se compararon con los registros almacenados en Moodle pertenecientes al conjunto de asignaturas de la titulación (3.615.751 logs) y con la media de todos los espacios virtualizados en la UCM.

Finalmente, los datos agregados fueron analizados con el programa IBM SPSS v25. El análisis estadístico realizado incluyó correlaciones paramétricas de Pearson y no paramétricas de Spearman, así como árboles de decisión mediante el criterio de corte CHAID (minimización de la significatividad del estadístico $\chi^{2}$ ) para relacionar rendimiento académico con segmentos de estudiantes con una actividad similar en CV.

\section{Resultados}

El comportamiento de los estudiantes en la asignatura virtualizada muestra una clara dependencia con el calendario académico a lo largo del curso. Todas las variables analizadas presentan picos de actividad coincidiendo con las fechas en que se realizaron los cuestionarios (líneas verticales grises) y de exámenes (líneas verticales rojas), con independencia del valor máximo alcanzado en cada una, como puede verse en la Fig 1. Los picos máximos de actividad registrada en un día son 1.906 visitas, 1.170 accesos a autoevaluaciones, 267 accesos a recursos, 179 a foros y 28 accesos a URLs. 

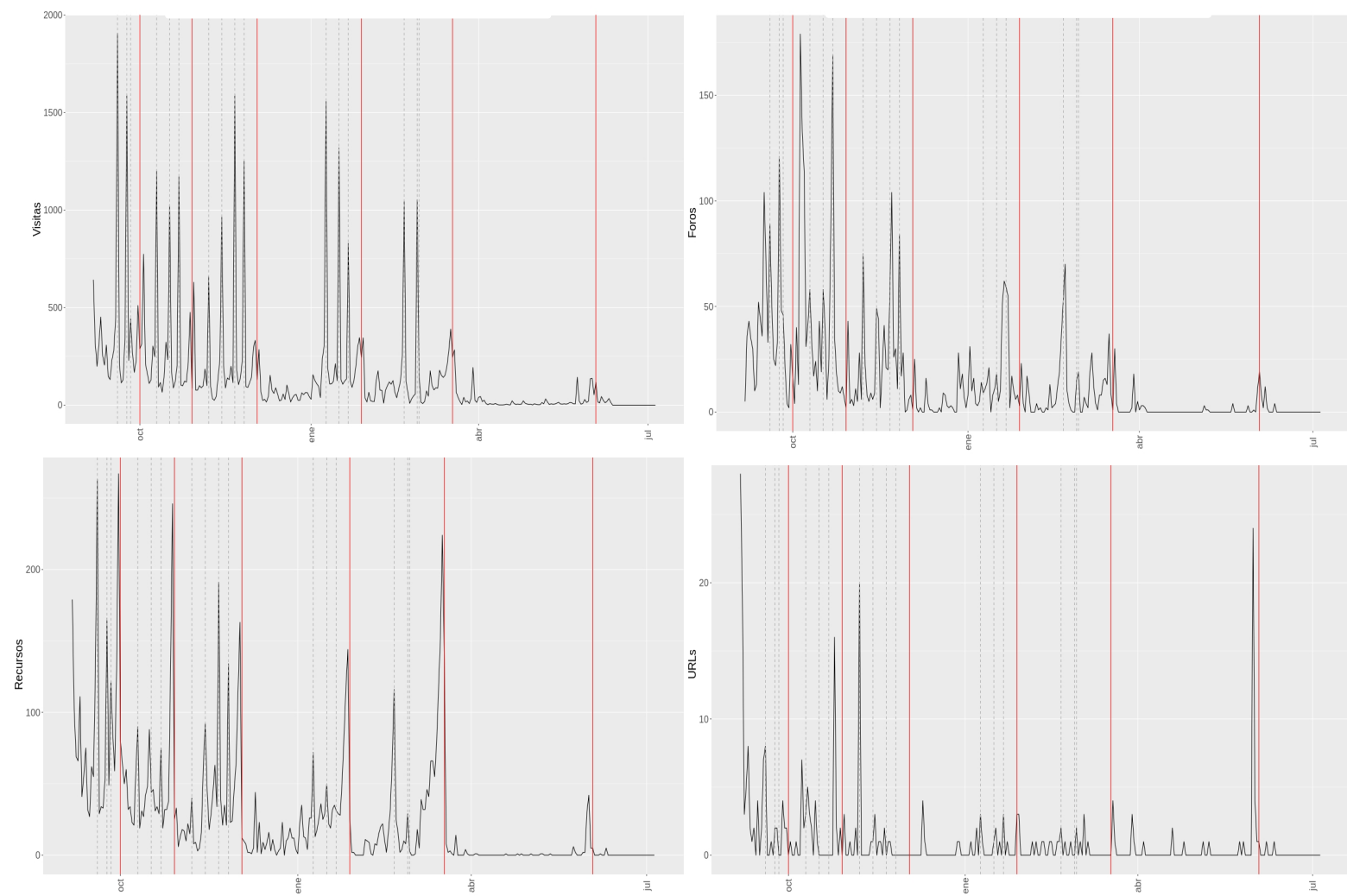

Fig. 1. Registros de la actividad de los estudiantes de OMH en el CV: visitas, accesos a foros, recursos y URLs

Los datos de acceso a la asignatura virtualizada por días de la semana reflejan una actividad media muy elevada todos los días de la semana, incluyendo los fines de semana, y muy superior a la media de la titulación (Fig. 2). 


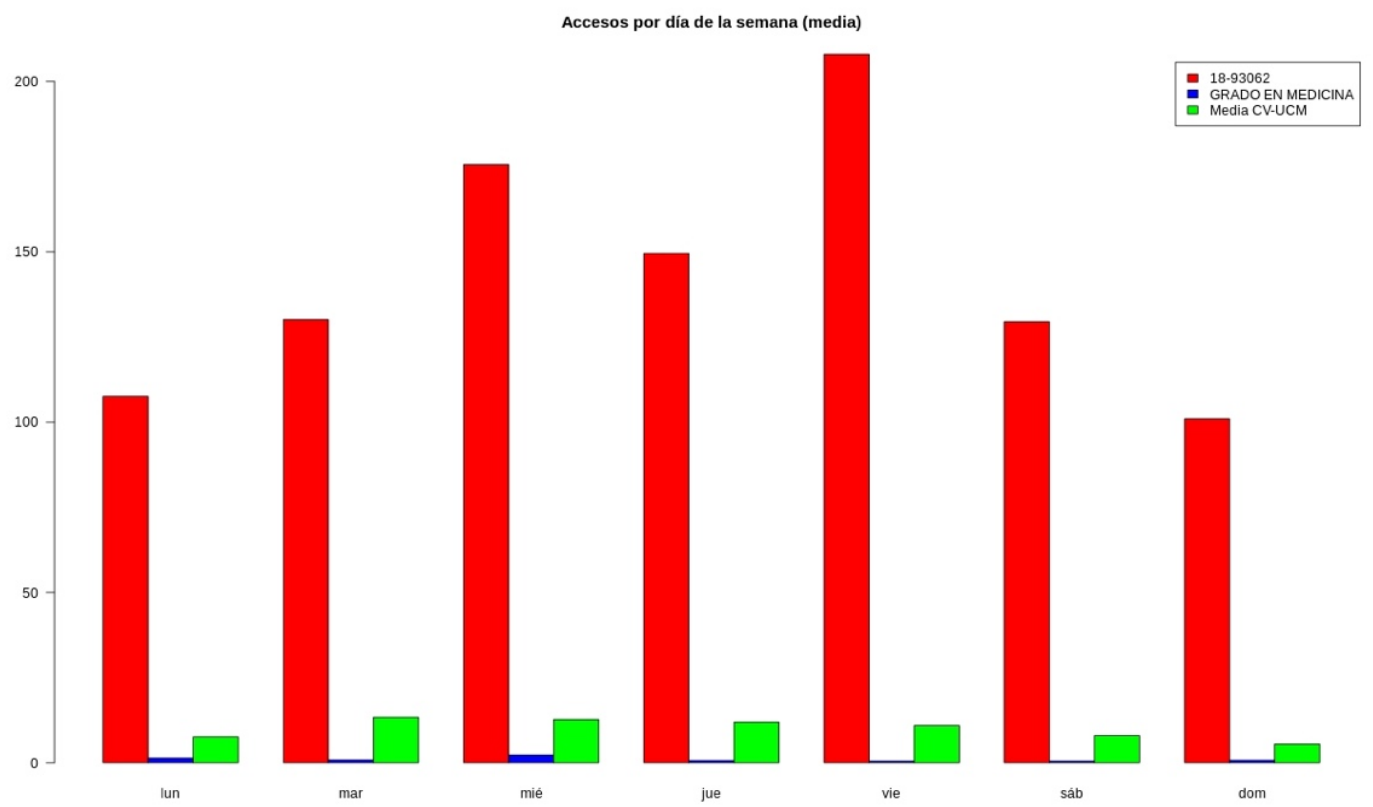

Fig. 2. Registros de los accesos medios semanales de los estudiantes de la asignatura OMH, del grado en Medicina y de la UCM, en el $C V$.

En cuanto a las horas del día, la Fig. 3 muestra que los valores de conexión máximos se encuentran por la tarde, entre las 17 y las 21 horas, en tanto que durante la mañana las horas de mayor conexión (11 a 13 horas) coinciden con el horario de clases teóricas (11:30 a 12:30 horas).

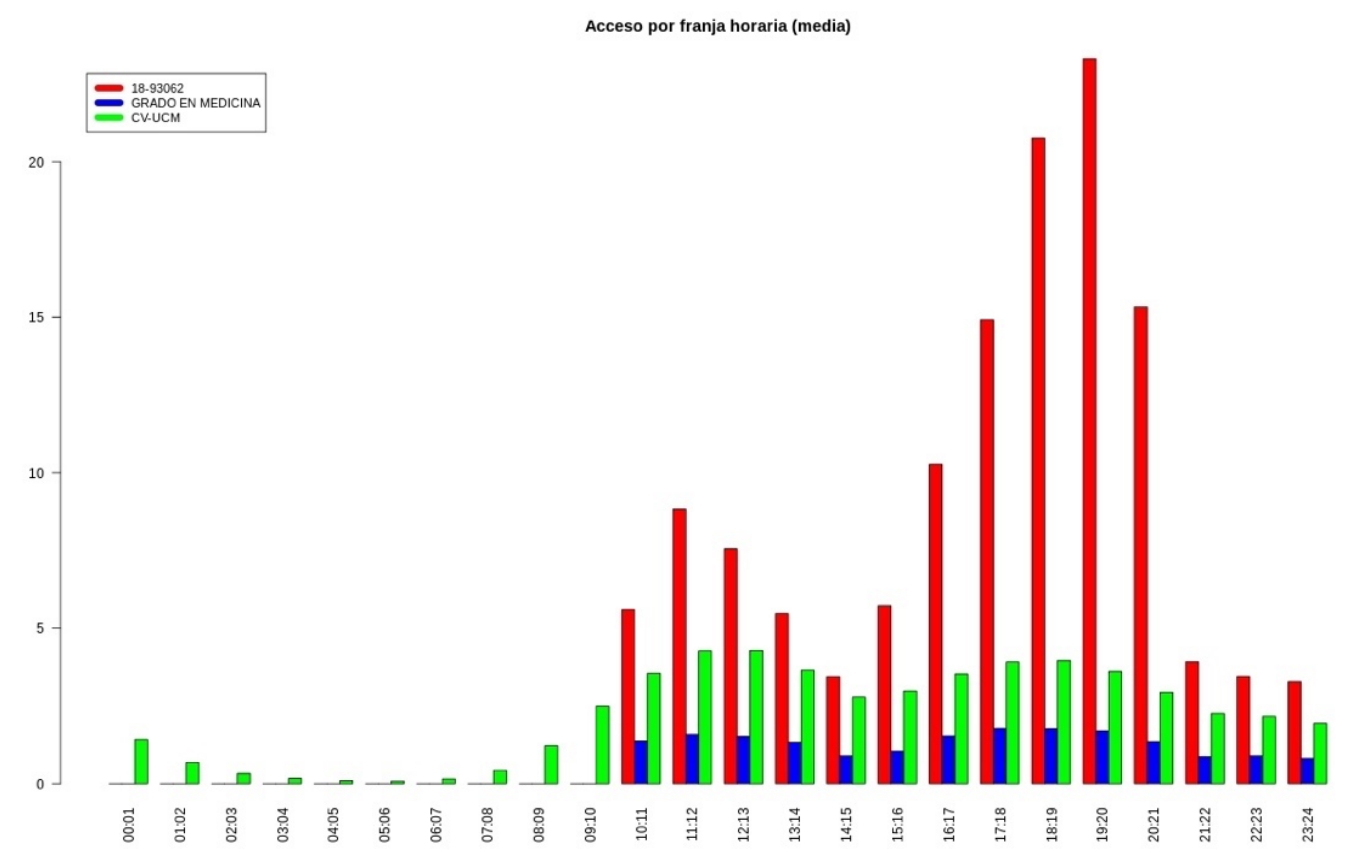

Fig. 3. Registros de los accesos medios diarios de los estudiantes de la asignatura OMH, del grado en Medicina y de la UCM, en el $\mathrm{CV}$. 
A la hora de analizar si el rendimiento académico está relacionado o no con el uso que hacen los estudiantes del CV, se establecieron 4 categorías según su nota final de teoría. La Tabla 1 recoge los valores de uso de cada variable analizada en el CV para cada categoría.

Tabla 1. Valores medios y desviación típica del uso medio que hace cada categoría de estudiantes según su nota final de teoría para cada variable analizada

\begin{tabular}{ccccc}
\hline & $<\mathbf{5}$ & $\mathbf{5 , 6 . 9 4 )}$ & $\mathbf{[ 6 . 9 5 , 8 . 5 4 )}$ & $>\mathbf{8 . 5 4}$ \\
\hline Número de estudiantes & 22 & 4 & 15 & 52 \\
Visitas & $333,86 \pm 185,41$ & $358,75 \pm 81,09$ & $513,07 \pm 302,44$ & $500,77 \pm 282,75$ \\
Recursos & $39,41 \pm 31,09$ & $109,5 \pm 45,27$ & $109,6 \pm 53,178$ & $97,19 \pm 43,52$ \\
Autoevaluaciones & $81,36 \pm 26,20$ & $89 \pm 14,856$ & $98,27 \pm 11,95$ & $91,92 \pm 11,13$ \\
URLs & $2,32 \pm 1,94$ & $0,5 \pm 0,58$ & $3,13 \pm 4,69$ & $2,73 \pm 3,64$ \\
Foros & $19,73 \pm 33,089$ & $15,5 \pm 18,73$ & $22,4 \pm 41,42$ & $56,33 \pm 80,90$ \\
\hline
\end{tabular}

Fuente propia

Los resultados obtenidos indican que cada categoría presenta un comportamiento diferente en el CV de acuerdo a los valores medios en las variables analizadas, lo cual se presenta en un gráfico de estrella multivariable de Kiviat (Fig. 4).

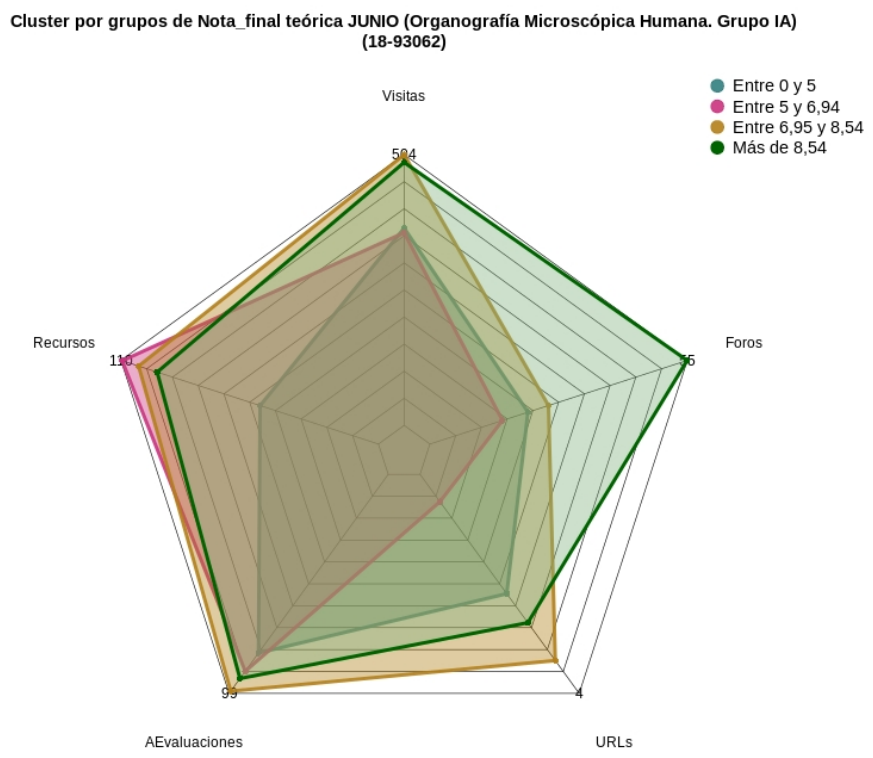

Fig.4. Comportamiento en el CV de los estudiantes según grupos por calificación final de teoría

(cc) BY-NC-ND 2020, Universitat Politècnica de València

Congreso In-Red (2020) 
En el grupo de estudiantes con calificaciones más altas (nota mínima 8,55) en la parte teórica en la convocatoria ordinaria (junio), destaca una media de accesos a los foros muy superior a la del resto de grupos (más del 50\% que la media de uso del siguiente grupo). Aunque aún no se ha podido desglosar la participación activa de la pasiva en foros, parece lógico deducir que este grupo de alumnos es el que más se ha podido beneficiar de la puntuación que se puede lograr por participación, si bien hay que señalar que existen otras formas distintas a las aportaciones en el foro general de dudas por las que se puede conseguir una puntuación extra adicional a la nota de los exámenes parciales. En el grupo de estudiantes con calificaciones entre 6,95 y 8,54, se observa un uso medio mayor de los enlaces a URLs. Es importante señalar que todos los estudiantes aprobados en junio superaron la parte teórica por parciales, esto es, quedaron exentos de realizar el examen final, mientras que el grupo que tuvo que hacer el examen final o bien no lo superó (nota inferior a 5) o bien no se presentó (grupo no incluido en la Fig. 4). El grupo de los suspensos muestra una media de accesos a cuestionarios y, en especial, a recursos claramente inferior a la de cualquiera de los grupos de aprobados por parciales (50\% inferior a la media de los tres grupos de aprobados).

La Fig. 5 muestra el árbol de decisión que compara el comportamiento en el CV de los alumnos que aprobaron la parte teórica por parciales (junio, color azul) y los que fueron al examen final y acabaron yendo a convocatoria extraordinaria (julio, color rojo). 


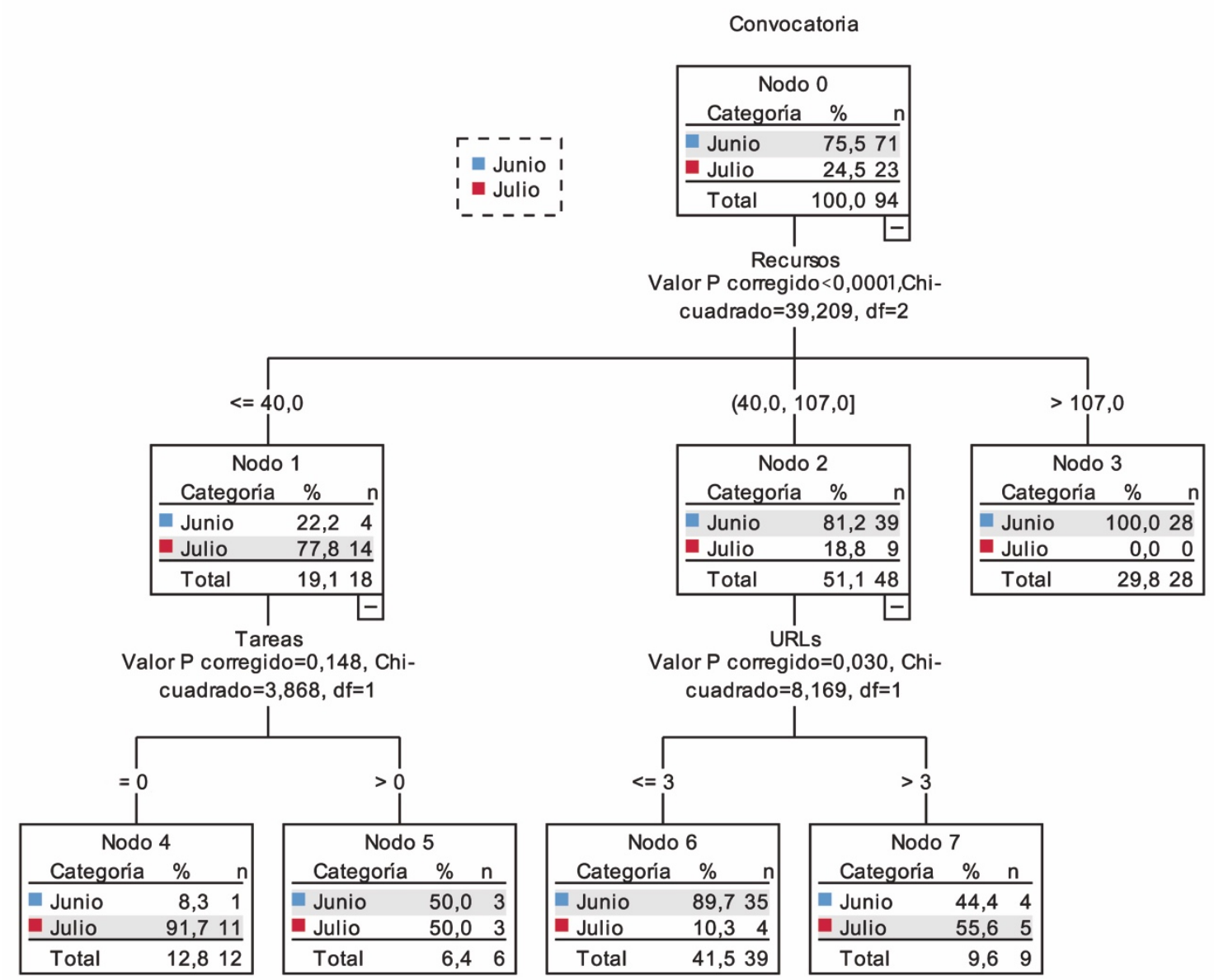

Fig. 5. Árbol de decisión que muestra las diferencias en el uso del CV entre aprobados por parciales (junio, azul) vs aprobados por examen final (julio, rojo). Método de crecimiento CHAID, con una proporción de casos clasificados incorrectamente de: 0,128 (error estándar: 0,034).

Mediante el método CHAID se obtienen tres nodos con diferencias estadísticamente significativas $(p<0,0001)$. A la derecha se observa el nodo 3 formado exclusivamente por alumnos que aprobaron por parciales, caracterizados porque el número de accesos a recursos fue superior a 107; en el centro, el nodo 2 está formado en un $81 \%$ por estudiantes que aprobaron por parciales, y que se caracterizan por un número de accesos a recursos entre 41 y 107; y a la izquierda, el nodo 1, está integrado mayoritariamente por alumnos que aprobaron en julio (78\%), y que se caracterizan por un número de accesos a recursos inferior a 41. Además de los tres nodos primarios se obtienen 4 nodos hijos más. El nodo 2 se desglosa en los nodos hijos 6 y 7, de acuerdo al acceso estadísticamente distinto a URLs $(\mathrm{p}=0,012)$ (de hasta en 3 ocasiones o en un número superior) mientras que del nodo 1 se obtienen los nodos hijos 4 y 5 , según hayan hecho o no una tarea voluntaria, si bien en este caso $p$ valor no es estadísticamente significativo $(p=0,135)$. 


\section{Discusión y conclusiones}

Esta investigación ha permitido conocer mejor el uso que hacen los estudiantes de los recursos y herramientas del CV y establecer relaciones entre el uso del CV y el rendimiento académico. El rendimiento académico es un concepto complejo que ha sido interpretado de distintas maneras pero, desde una perspectiva operativa, se define como la nota o calificación media obtenida durante el periodo universitario que cada alumno haya cursado (Tejedor, 1998). El rendimiento académico es un indicador directo de la calidad de la enseñanza y como tal está plenamente asumido por los responsables universitarios y la sociedad en general (Escudero, 2000).

Distintos autores han estudiado cómo influyen la motivación, los hábitos de estudio, la formación previa o las metodologías docentes y de aprendizaje en el rendimiento académico de los estudiantes de Histología (Bloodgood, 2012; Campos-Sánchez et al., 2012 y 2014; Selvig et al., 2015). Este trabajo demuestra que los resultados académicos de los estudiantes de OMH están estrechamente condicionados por el uso que hayan hecho de las herramientas y recursos del CV, lo cual está en línea con los hallazgos en otras disciplinas (Yu y Jo, 2014; Cerezo et al., 2016; Młynarska et al., 2016; Jenaro et al., 2018; Cantabella et al., 2019). Los resultados muestran que el alumnado de $\mathrm{OMH}$ accede al CV asiduamente, siendo su actividad mucho mayor a la media de la titulación y en clara dependencia con las actividades programadas, como se ha apuntado en estudios anteriores (Plendl et al., 2009; Álvarez et al., 2020a, b).

La reforma del plan Bolonia ha supuesto la implantación de la evaluación continua. Ello junto con la introducción de metodologías docentes mixtas (blended learning) ha propiciado que el alumnado de la $\mathrm{OMH}$ se vuelque en aprobar la asignatura por parciales para lo cual la asignatura virtualizada representa un gran apoyo (87\% consideró al CV como Importante o Muy importante en la encuesta final, datos no publicados). Resulta ilustrativo de la presión a la que los alumnos de este curso están sometidos (gran número de exámenes parciales a lo largo del curso, exámenes consecutivos el mismo día de $\mathrm{AH}, \mathrm{FH}$ y $\mathrm{OMH}$, ninguna semana de descanso) el hecho de que todos los que no quedaron exentos de realizar el examen final teórico lo suspendieran en la primera convocatoria. La profesora favorece el aprendizaje autónomo y colaborativo mediante actividades realizadas en clase, como la resolución de casos clínicos con base histológica y la elaboración de mapas conceptuales, y fuera de clase, mediante cuestionarios programados para que el estudio sea continuado y no solo previo a las fechas de examen, así como mediante el planteamiento y resolución de dudas o la contribución con noticias o artículos científicos con interés histológico. Aunque la participación en el foro general y en los cuestionarios es vista inicialmente por el alumnado como algo necesario para aprobar cada parcial, lo cierto es que finalmente redunda no solo en un porcentaje de aprobados considerablemente alto en la convocatoria ordinaria (75,5\%) sino en elevar las notas que los estudiantes obtienen ( $73 \%$ con nota final de teoría sobresaliente). Los resultados indican que un mayor compromiso y motivación se traducen en un uso significativamente mayor del CV y ello conduce a calificaciones más altas, y por el contrario, los estudiantes que suspenden muestran un menor uso del CV.

La motivación es particularmente importante en la histología por tratarse de una materia conceptualmente compleja, en la que es importante integrar correctamente imágenes y conceptos, y en la que no siempre los estudiantes alcanzan a comprender la importancia de su aprendizaje de cara a sus aplicaciones a lo largo de su formación y a largo plazo (Campos-Sánchez et al., 2014). La actividad registrada en los LMS puede 
proporcionar una medida indirecta válida del compromiso y motivación del alumnado (Motz et al., 2019). Así pues, un uso escaso o limitado del CV puede ser un indicador que alerte al profesorado y le mueva a tutorizar de forma individualizada y a tratar de estimular a los estudiantes poco motivados, al igual que la detección temprana del absentismo ha sido una forma de identificar alumnos en riesgo de abandono o de fracaso en la materia (Espada, 2008; Sacristán-Díaz et al., 2012; Selvig et al., 2015, Álvarez, 2016). Entendemos, pues, que el uso de las TIC en el mundo educativo ofrece nuevas posibilidades que pueden ayudar a la implicación activa de los estudiantes y a la personalización de la enseñanza.

Como conclusiones finales de este trabajo debemos señalar que cada grupo de estudiantes de $\mathrm{OMH}$, de acuerdo a su nota final teórica, tuvo compartamientos diferenciales y que hay claras diferencias entre el grupo de suspensos, el de notables y el de sobresalientes. Asímismo, se observó que entre estudiantes con bajo rendimiento el uso medio de recursos y cuestionarios fue claramente inferior mientras que los estudiantes con mejores rendimientos son más activos en los foros o el uso de las URLs. Resulta importante, pues, trabajar las herramientas del $\mathrm{CV}$, hacer un uso avanzado del $\mathrm{CV}$ porque promueve en una mayor participación y un mejor aprendizaje de los alumnos.

\section{Agradecimientos}

Los autores agradecen el apoyo recibido por el Vicerrectorado de Tecnologías de la Información y la Oficina eCampus de la UCM.

\section{Referencias}

ÁLVAREZ VÁZQUEZ, M.P. (2016). “Absentismo universitario en alumnos de grado ¿motivados? ” en Bolarín Martínez, M.J.; Porto Currás, M. y García Hernández, L. Evaluación e identidad del alumnado en Educación Superior. Murcia: Universidad de Murcia, pp. 719-724.

ÁLVAREZ VÁZQUEZ, M.P., ÁLVAREZ-MÉNDEZ A.M., BRAVO-LLATAS, M.C., CRISTÓBAL BARRIOS, J. y ANGULO CARRERE, T. (2020a). "Tipologías de estudiantes de Fisioterapia según el uso que hacen del campus virtual" en Revista Innovación Docente Universitaria vol. 12, pp.74-81.

ÁlVAREZ VÁZQUEZ, M.P., ÁlVAREZ-MÉNDEZ A.M., ANGULO CARRERE, T., CRISTÓBAL BARRIOS, J. y BRAVO-LLATAS, M.C. (2020b) 'Learning analytics in human histology reveals different student' clusters and different academic performance". En: INTED2020 Proceedings 14th International Technology, Education and Development Conference. Valencia, España. IATED. 66-72.

BLOODGOOD, R. A. (2012). "Active learning: A small group histology laboratory exercise in a whole class setting utilizing virtual slides and peer education" en Anatomical Sciences Education, vol. 5, pp. 367-373.

CAMPOS-SÁNCHEZ, A., MARTÍN-PIEDRA, M.A., CARRIEL, V., GONZÁLEZ-ANDRADES M., GARZÓN, I., SÁNCHEZ-QUEVEDO, M.C. y ALAMINOS., M. (2012). "Reception learnign and self-discovery learning $\mathrm{n}$ histology: Students' perceptions and their implications for assessing the effectiveness of different learning modalities" en Anatomical Sciences Education vol. 5, pp. 273-280. 
CAMPOS-SÁNCHEZ, A., LÓPEZ-NUÑEZ, J.A., CARRIEL, V., MARTÍN-PIEDRA, M.A., SOLA, T. y ALAMINOS., M. (2014). "Motivational component profiles in university students learning histology : a comparative study between genders and different health science curricula" en BMC Medical Education vol. 14:46.

CANTABELlA, M., MARTÍNEZ-ESPAÑA, R., AYUSO, B., YAÑEZ J.A., y MUÑOZ, A. (2019). “Analysis of student behavior in learning management systems through a Big Data framework" en Future Generation Computer Systems vol. 90, pp. 262-272.

CASEY, K. y GIBSON, P. (2010) “(m)Oodles of Data. Mining Moodle to understand Student Behaviour”. En: ICEP10 International Conference on Engaging Pedagogy 2010. Maynooth, Irlanda.

CEREZO, R., SÁNCHEZ-SANTILLÁN, M., PAULE-RUIZ, M.P. y NUÑEZ, J.C. (2016). “Students’ LMS interaction patterns and their relationships with achievement : A case study in higher education" en Computers \& Education vol. 96, pp.42-54.

CHAPARRO PELÁEZ, J., IGLESIAS PRADAS, S. y PASCUAL MIGUEL, F. (2010). "Uso del registro de actividad de Moodle para un estudio del rendimiento académico de alumnos en entornos en línea y presencial”. En 4th International Conference on Industrisl Engineering and Industrial Management, XIV Congreso de Ingenieria de Organización. Donostia San Sebastian, España. pp. 753-760.

DANIEL, B. (2015). "Big data and analytics in higher education : opportunities and challenges" en British Journal of Educational Technology vol. 46, pp.904-920.

ESCUDERO, T. (2000). "La evaluación y mejora de la enseñanza en la universidad: otra perspectiva" en Revista de investigación Educativa, vol. 18, pp. 405-416.

FERRERO, R. y LÓPEZ J.L. (2018). Data science ¿Qué es R software? En Máxima Formación. https://www.maximaformacion.es/blog-dat/que-es-r-software/ [Consulta: 20 de febrero 2020].

GARCÍA-PEÑALVO, F.J., HERNÁNDEZ-GARCÍA, A., CONDE, M.A., FIDALGO-BLANCO, A., SEINECHALUCE, M.L., ALIER, M., LLORENS-LARGO, F. y IGLESIAS-PRADAS, S. (2015). "Mirando hacia el futuro: Ecosistemas tecnológicos de aprendizaje basados en servicios". En: CINAIC2015 III Congreso Internacional sobre Aprendizaje, Innovación y Competitividad. Madrid, España. pp.553-558.

JENARO RÍO, C., CASTAÑO CALlE, R., MARTín PASTOR, M.E. y FLORES ROBAINA, N. (2018). "Rendimiento académico en educación superior y su asociación con la participación activa en la paltaforma Moodle" en Estudios sobre Educación vol. 34: pp. 277-198.

LLORENS LARGO, F. (2014). "Campus virtuales: de gestores de contenidos a gestores de metodologías" en RED, Revista de Educación a Distancia vol, 42.

LONG, P. y SIEMENS, G. (2011). "Penetrating the fog: Analytics in learning and education" en EDUCAUSE Review vol. 46, pp.31-40.

MAS TORELLÓ, O. (2011). "El profesor universitario: sus competencias y formación” en Profesorado. Revista de Currículum y Formación de Profesorado vol. 15, pp. 195-211.

MAYER-SCHÖNBERGER, V. y CUKIER, K. (2013). Big Data. La revolución de los datos masivos. Madrid: Turner Publicaciones SL.

MŁYNARSKA, E., GREENE, D. y CUNNINGHAM, P. (2016). "Indicators of Good Student Performance in Moodle Activity Data” en arXiv:1601.02975v1 [cs.CY].

MOTZ, B., QUICK, J., SCHROEDER, N., ZOOK, J. y GUNKEL, M. (2019). "The validity and utility of activity logs as a measure of student engagement". In LAK19 The 9th International Learning Analytics \& Knowledge Conference. Tempe, EE.UU. pp. 300-309.

OLLÉ, J. (2019). “¿Cómo seleccionar y utilizar el mejor software para poder adaptarte a la era de los datos y ser único en tu sector? en Conceptos Claros en https://conceptosclaros.com/software-analisis-datos/ [Consulta: 12 de febrero 2020].

PAULSEN, M. F. (2003). "Experiences with learning management systems in 113 European institutions" en Journal of Educational Technology \& Society, vol.6, pp.134-148. 
PLENDL, J., BAHRAMSOLTANI, M., GEMEINHARDT, O., HUNIGEN, H., KABMEYER, S. y JANCZYK, P. (2009). "Active participation instead of passive behaviour opens up new vistas in education of veterinary anatomy and histology" en Anatomy Histology and Embriology vol. 38, pp.355-360.

ROMERO, C., LÓPEZ, M.I., LUNA, J.M. y VENTURA, S. (2013). "Predicting students'final perfomance from participation in on-line discussion forums" en Computers \& Education vol. 68, pp.458-472.

SACRISTÁN-DÍAZ, GARRIDO-VEGA, P., GONZÁLEZ-ZAMORA, M.M. y ALFALLA-LUQUE R. (2012). “¿Por qué los alumnos no asisten a clase y no se presentan a los exámenes? Datos y reflexiones sobre absentismo y abandono universitarios" en Working Papers on Operations Management vol. 3, pp.101-112.

SALINAS, J. (2004). "Innovación docente y uso de las TIC en la enseñanza universitaria” en Revista Universidad y Sociedad del Conocimiento [artículo en línea]. UOC. vol. 1, n ${ }^{\mathrm{o}}$ 1. [Fecha de consulta: 07/02/2020]. $<$ http://www.uoc.edu/rusc/dt/esp/salinas1104.pdf $>$

SELVIG, D., HOLADAY, L.W., PURKISS, J. y HORTSCH, M. (2015). “Correlating students' educational background, studdy habits, and resource usage with learning success in medical histology" en Anatomical Sciences Education vol. 8, pp. 1-11.

TEJEDOR, F.J. (Coord) (1998) Los alumnos de la Universidad de Salamanca. Características y Rendimiento Académico. Ediciones Universidad de Salamanca. 244 pp.

TORRES-PORRAS, J., ALCÁNTARA, J. y RUBIO, S. (2018). "Virtual platforms use: a useful monitoring tool" en Revista de Educación Mediática y TIC vol. 7, pp. 242-255.

UNIVERSIDAD COMPLUTENSE DE MADRID. FACULTAD DE MEDICINA (2019). Guía del Grado en Medicina. Nivel de Máster universitario. 2019-2020. Disponible en https://medicina.ucm.es/data/cont/media/www/pag-17227/GUÍA\%20MEDICINA\%202019-2020.\%20FINAL\%20septiembre\%202019.pdf [Consulta: 1 febrero de 2020]

YU, T. y JO, I. (2014). "Educational technology approach toward learning analytics: relationship between student online behavior and learning performance in higher education". In Proceedings of the fourth International Conference on Learning Analytics and Knowledge. Indianapolis, EE.UU. pp. 269-270.

ZAMBRANO, W.R., MEDINA, V.H. y GARCÍA, V.M. (2010). "Nuevo rol del profesor y del estudiante en la educación virtual” en Dialéctica Revista de Investigación vol. 26, pp. 51-62. 